\title{
Stability of orthodontic treatment and dental extractions
}

David Normando1', Guilherme Janson²

"Success is the ability to go from failure to failure without losing your enthusiasm" Winston Churchill, British politician and writer.

Today's orthodontics has substantially reduced the need for extractions. One of the reasons for this change in approach is based on the findings of studies published in the early 1980s by researchers at the University of Washington, who reported similar rates of teeth alignment relapse in cases treated with or without premolar extractions. ${ }^{1,2}$ Thus, it was reported that the decision to extract teeth would not improve the chances of increased long term orthodontic treatment stability. However, Little et $\mathrm{al}^{1,2}$ focused their studies on the changes that occurred in teeth alignment. If one glimpses beyond crowding, an analysis of the literature will undoubtedly reveal that this assumption may be wrong, and the reverberating truth may in the future damage our ears, as it will certainly damage the patients' teeth.
Regarding the stability of sagittal correction, more specifically in the treatment of Class II malocclusion, there used to be a dogma, still recurrent in Orthodontics, that the molars should be finished in a Class I relationship to provide greater treatment stability. ${ }^{3}$ Nevertheless, this assertion finds no support when treatment stability is analyzed comparatively, i.e., between protocols involving extractions of two maxillary premolars and nonextraction protocols. ${ }^{4} \mathrm{~A}$ recent systematic review indicated as inconclusive whether or not stability would be achieved to a greater or lesser degree if a Class II treatment were performed with or without extractions. ${ }^{5}$ On the other hand, there seems to be evidence, albeit relatively scant, indicating that cases with Class III malocclusion treated compensatorily with extractions of lower teeth are more stable than nonextraction cases. ${ }^{6,7}$

\footnotetext{
${ }^{1}$ Adjunct Professor of Orthodontics, School of Dentistry, Universidade Federal do Pará (UFPA). Coordinator of the Graduate program in Dentistry at UFPA and the Specialization Course in Orthodontics at ABO-Pará.

${ }^{2}$ Full Professor of Orthodontics at Bauru School of Dentistry, Universidade de São Paulo (FOB-USP).
} 
Even if extractions appear to lessen the risk of relapse of compensatory orthodontic treatment of Class III malocclusion, the same results have been yielded by studies examining stability of non-surgical treatment of anterior open bite. Clinical studies have revealed that stability seems to improve when treatment in the permanent dentition is performed with extractions, be it in adolescents ${ }^{8}$ or in adults. ${ }^{9}$ It has been speculated that stability is improved in extraction treatments precisely because two mechanisms help in closing the anterior open bite, i.e., the "drawbridge principle," and the mesialization of posterior teeth. ${ }^{8}$

But why should we broach this subject again? Thanks to the advent and popularization of skeletal anchorage there has been a vast increase in treatment approaches whereby sagittal skeletal errors are compensated by en mass distalization of the maxillary teeth in Class II cases, or the mandibular teeth, in Class III cases. Furthermore, the option of intruding the posterior teeth in cases of anterior open bite with the help of skeletal anchorage devices has been increasingly gaining momentum. These treatment protocols boast the unique advantage of reducing the indication of extractions. Conversely, if the prospect of a lower chance of relapse of sagittal and vertical changes (anterior open bite) in extraction cases is true, it is highly likely that we are raising the risk of relapse with the aid of skeletal anchorage, in nonextraction cases.

At this point, when opting for such a treatment protocol, it would be wise to inform the patient that we are embarking on a treatment with a higher risk of relapse. At least for now, while we wait for a scientific answer.

David Normando, Guilherme Janson
1. Little RM, Wallen TR, Riedel RA. Stability and relapse of mandibular anterior alignment-first premolar extraction cases treated by traditional edgewise orthodontics. Am J Orthod. 1981 Oct;80(4):349-65.

2. Little RM, Riedel RA, Artun J. An evaluation of changes in mandibular anterior alignment from 10 to 20 years postretention. Am J Orthod Dentofacial Orthop. 1988 May:93(5):423-8.

3. Mailankody J. Enigma of Class II molar finishing. Am J Orthod Dentofacial Orthop. 2004 Dec;126(6):A15-6; author reply A16-7.

4. Janson G, Camardella LT, Araki JD, de Freitas MR, Pinzan A. Treatment stability in patients with Class II malocclusion treated with 2 maxillary premolar extractions or without extractions. Am J Orthod Dentofacial Orthop. 2010 July;138(1):16-22.

5. Maniewicz Wins S, Antonarakis GS, Kiliaridis S. Predictive factors of sagittal stability after treatment of Class II malocclusion. Angle Orthod. 2016 Nov; 86(6):1033-41.
6. Yoshizumi J, Sueishi K. Post-treatment stability in Angle Class III sases. Bull Tokyo Dent Coll. 2016:57(1):29-35.

7. Zimmer B, Schenk-Kazan S. Dental compensation for skeletal Class III malocclusion by isolated extraction of mandibular teeth. Part 1: Occlusal situation 12 years after completion of active treatment. J Orofac Orthop. 2015 May:76(3):251-64

8. Janson G, Valarelli FP, Beltrão RT, de Freitas MR, Henriques JF. Stability of anterior open-bite extraction and nonextraction treatment in the permanent dentition. Am J Orthod Dentofacial Orthop. 2006 June:129(6):768-74.

9. Medeiros RB, Araújo LFC, Mucha JN, Motta AT. Stability of open-bite treatment in adult patients: A systematic review. J World Fed Orthod. 2012;1(3):e97-101. 